\title{
XMM-Newton discovery of a Compton-thick AGN in the GPS galaxy Mkn 668
}

\author{
M. Guainazzi ${ }^{1}$, A. Siemiginowska ${ }^{2}$, P. Rodriguez-Pascual ${ }^{1}$, and C. Stanghellini ${ }^{3}$ \\ 1 XMM-Newton Science Operation Center, VILSPA, ESA, Apartado 50727, 28080 Madrid, Spain \\ 2 Harvard-Smithsonian Center for Astrophysics, 60 Garden St., Cambridge, MA 02138, USA \\ ${ }^{3}$ Istituto di Radioastronomia CNR, Noto, Italy
}

Received 12 January 2004 / Accepted 3 March 2004

\begin{abstract}
We report the XMM-Newton discovery of the first Compton-thick obscured AGN in a Broad Line Radio Galaxy, the Gigahertz Peaked-Spectrum source Mkn 668 (OQ+208). The remarkably flat 2-10 keV X-ray spectrum (observed photon index, $\Gamma \simeq 0.7$ ), with a prominent iron $\mathrm{K}_{\alpha}$ fluorescent emission line, is a clear signature of a Compton-reflection dominated spectrum. Mkn 688 represents a remarkable example of discrepancy between X-ray spectral properties and optical classification, as its optical spectrum is characterized by broad and asymmetric Balmer lines. The obscuring matter is constrained to be located within the radio hotspots, in turn separated by about $10 \mathrm{pc}$. If the jets are piercing their way through a Compton-thick medium pervading the nuclear environment, one could be largely underestimating the radio activity dynamical age determined from the observed hotspot recession velocity. The soft X-ray spectrum is dominated by a much steeper component, which may be due to nuclear continuum electron scattering, or inverse Compton of the - remarkably large - far infrared emission. Soft X-rays are suppressed by a further Compton-thin $\left(N_{\mathrm{H}} \sim 10^{21} \mathrm{~cm}^{-2}\right)$ absorbing system, that we identify with matter responsible for free-free absorption of the radio lobes.
\end{abstract}

Key words. galaxies: individual: Mkn 668 - galaxies: jets - galaxies: nuclei - galaxies: Seyfert - X-ray: galaxies

\section{Introduction}

Gigahertz Peaked-Spectrum (GPS) radio sources are a class of compact (10-100 mas; 10-100 parsecs) radio sources, characterized by a simple convex radio spectrum peaking around $1 \mathrm{GHz}$ (see O'Dea et al. 1998 for a review). Together with the less compact and steeper Compact Steep-Spectrum (CSS) sources, they constitute a sizable fraction of the $5 \mathrm{GHz}$ selected sources, probably as large as 40\% (O’Dea 1998).

In the ROSAT All Sky Survey, GPS/CSS quasars exhibited a detection rate 3 times lower than radio-loud quasars of comparable power (Baker et al. 1995). The explanation for their X-ray weakness is still matter of debate. They may represent an intrinsically X-ray weak population of radio-loud Active Galactic Nuclei (AGN); alternatively, their flux in the soft X-ray band may be suppressed by matter heavily obscuring the active nucleus. The first pointed observations with ROSAT suggested that X-ray absorption in GPS/CSS quasars may be common (Elvis et al. 1994). The first hard X-ray measurements of GPS galaxies needed to await ASCA, which revealed column densities of the order of $10^{22} \mathrm{~cm}^{-2}$ (O'Dea et al. 2000; Guainazzi et al. 2000). However, the number of GPS galaxies

Send offprint requests to: M. Guainazzi,

e-mail: mguainaz@xmm.vilspa.esa.es for which X-ray spectroscopic measurements are available remains low.

The X-ray properties of GPS galaxies may have immediate consequences for our understanding of their nature, and, in turn, on the evolution of large-scale radio structures in the universe. The detection of hotspot proper motions in a handful of Compact Symmetric Objects (Polatidis \& Conway 2003), corresponding to dynamical ages $\sim 10^{2}-10^{3}$ years, suggests that at least some GPS galaxies should represent an infancy stage of radio galaxies proper (Phillips \& Mutel 1982; Carvalho 1985; Fanti et al. 1995; Readhead et al. 1996). However, there is convincing evidence for the presence of large amount of matter in the core of GPS sources (de Vries et al. 1998; Pihlström et al. 2003; Snellen et al. 2002). It is still unclear whether this matter has the average density $\left(\sim 1-10 \mathrm{~cm}^{-3}\right)$ required to ensure the permanent confinement of the radio structure (De Young 1993), and to prevent ("frustrate") its full development into a Fairnoff-Railey (FR) II, or, most likely, into a FR I galaxy (O'Dea et al. 2000). X-ray probing of the circumnuclear environment may represent an important clue.

Thanks to the unprecedented collecting area of its optics, XMM-Newton (Jansen et al. 2001) is the ideal observatory to perform high-sensitivity, moderate-resolution spectroscopy of weak $\left(10^{-13} \mathrm{erg} \mathrm{cm}^{-2} \mathrm{~s}^{-1}\right) \mathrm{X}$-ray sources. An observation 
program is being carried out, aiming at studying the properties of the circumnuclear gas in a sizable sample of GPS galaxies. In this paper, we report on the observation of Mkn 668, which constitutes to date one of the very few hard X-ray measurements of a GPS galaxy. XMM-Newton unveiled the first, to our knowledge, Compton-thick AGN in a broad-line radio galaxy. Mkn 668 (OQ+208, 1404+286) is one of the closest known GPS galaxy ( $z=0.0077$; Stanghellini et al. 1993). The host galaxy shows signs of tidal distortion (Stanghellini et al. 1993), suggesting a recent merging event. Optically classified as a Seyfert 1 (Blake et al. 1970; Marziani et al. 1993), its radio morphology exhibits two radio lobes along the NE:SW direction at the approximate distance of $10 \mathrm{pc}$. Marginal evidence for a radio core closer to the former and brighter lobe were recently reported by Lister (2003). The whole radio structure is embedded in a large diffuse halo of diameter $\simeq 30 \mathrm{kpc}$ (de Bruyn 1990). The hotspots separate with an apparent velocity of 30-60 $\mu$ as $\mathrm{yr}^{-1}$ (Stanghellini et al. 2002; Lister 2003; Barone 2003). This identifies Mkn 668 as a potential "young" radio source, with an estimated dynamical age of $\simeq 100-200$ years. Kameno et al. (2000) fit the longwavelength radio cutoff with free-free absorption by matter embedding the radio structure. They claimed that thermal emission from the absorbing gas is responsible for the soft X-ray emission detected by ROSAT (Zhang \& Marscher 1994).

An ASCA observation of Mkn 668 (Guainazzi et al. 2003) unveiled a very flat X-ray spectrum, together with a possible bright (Equivalent Width, $E W \simeq 900 \mathrm{eV}$ ) $\mathrm{K}_{\alpha}$ fluorescent iron line. These results prompted the XMM-Newton observation, which is the main topic of this paper.

In this paper, energies are quoted in the source frame; errors are quoted at the $1-\sigma$ level for the count rates, and at the $90 \%$ confidence level for one interesting parameter for fit parameters and derived quantities; $H_{0}=70 \mathrm{~km} \mathrm{~s}^{-1} \mathrm{Mpc}^{-1}$; and $q_{0}=0.5$. At the Mkn 668 redshift, $1^{\prime \prime} \simeq 1.5 \mathrm{kpc}$.

\section{XMM-Newton observation}

XMM-Newton observed the sky region around Mkn 668 on January 31, 2003. In this paper only results from the EPIC cameras (pn; Strüder et al. 2001; MOS, Turner et al. 2001) will be discussed, as Mkn 668 is too weak to be detected by the highresolution spectroscopy cameras (RGS). Data were reduced with SAS v5.4.1 (Jansen et al. 2001), using the most updated calibration files available at the moment the reduction was performed (March 2003). Standard procedures were followed for the EPIC data reduction and analysis, as detailed in the $S A S$ User's Guide (Loiseau 2003). In particular, scientific products were accumulated combining single and double (to quadruple) pn (MOS) events (this is most appropriate for moderately weak sources to enhance the signal-to-noise ratio); background products were extracted from regions belonging to the same CCD as Mkn 668 and free form contaminating sources; intervals of high particle background were removed, applying standard thresholds to the $E>10 \mathrm{keV}$, single event field-of-view light curves ( 1 and 0.35 counts per second for the pn and the MOS cameras, respectively). After screening, the total exposure time was 13.2 and $15.5 \mathrm{ks}$ for the pn and MOS cameras,
Table 1. Position and count rate (CR) of the sources detected by the XMM-Newton EPIC cameras within 3' from the optical nucleus of Mrk 668, and having a signal-to-noise ratio larger then 5.

\begin{tabular}{lcc}
\hline \hline$\alpha_{2000}$ & $\delta_{2000}$ & $\begin{array}{c}0.5-10 \mathrm{keV} \\
\mathrm{CR}\left(10^{-2} \mathrm{~s}^{-1}\right)\end{array}$ \\
\hline $14^{\mathrm{h}} 07^{\mathrm{m}} 00.3^{\mathrm{s} a}$ & $+28^{\circ} 27^{\prime} 14^{\prime \prime} a$ & $14.5 \pm 0.4^{a}$ \\
$14^{\mathrm{h}} 06^{\mathrm{m}} 57.4^{\mathrm{s}}$ & $+28^{\circ} 28^{\prime} 5^{\prime \prime}$ & $1.41 \pm 0.19$ \\
$14^{\mathrm{h}} 07^{\mathrm{m}} 04.4^{\mathrm{s}}$ & $+28^{\circ} 29^{\prime} 37^{\prime \prime}$ & $1.03 \pm 0.18$ \\
\hline
\end{tabular}

${ }^{a}$ Mkn 668.

respectively. The event lists of the two MOS cameras were merged before extracting any scientific products. Version 5.1 of the LHEASOFT package was employed for the scientific analysis described hereinafter.

\subsection{Imaging}

In the EPIC field-of-view image a source is clearly detected close to the nominal boresight positions. In the pn, $\alpha_{2000}=$ $14^{\mathrm{h}} 07^{\mathrm{m}} 00.3^{\mathrm{s}}, \delta_{2000}=+28^{\circ} 27^{\prime} 14^{\prime \prime}$. The $0.5-10 \mathrm{keV}$ count rates are $(8.6 \pm 0.4)$, and $(6.0 \pm 0.3) \times 10^{-2} \mathrm{~s}^{-1}$ in the pn and MOS cameras, respectively. The best-fit centroid is $1.5^{\prime \prime}$ distant from the galaxy core optical position, consistent with typical XMM-Newton attitude reconstruction accuracy. There is no evidence for intrinsic extension beyond the moderately broad XMM-Newton mirror Point Spread Function (6" Full Width Half Maximum; FWHM). Two additional sources are detected at a signal-to-noise ratio larger than 5 within $3^{\prime}$ from the Mrk 668 nucleus (i.e. within the typical ASCA aperture; see Sect. 3.1). Their position and count rates are reported in Table 1. The sum of their count rates amounts at $29 \pm 10 \%$ and $<11 \%$ of Mrk 668 count rate in the $0.5-2$ and $2-10 \mathrm{keV}$ energy bands, respectively.

\subsection{Spectral analysis of the nuclear emission}

Mkn 668 nucleus spectra and light curves were extracted from regions of $40^{\prime \prime}$ and $25^{\prime \prime}$ radius for the pn and the MOS, respectively. Total source counts are $880 \pm 30$ and $620 \pm 20$ in the pn and MOS, respectively, $15 \%$ and $11 \%$ of which are due to background. No clear evidence for variability of the Mkn 668 nucleus flux is seen during the XMM-Newton observation. A fit with a constant function on the pn $1024 \mathrm{~s}$ binned light curves in the $0.5-2 \mathrm{keV}$ and $2-10 \mathrm{keV}$ energy bands yields $\chi_{v}^{2}=0.81$ and $\chi_{v}^{2}=1.01$, respectively. We will therefore focus in the following on the time-averaged spectra only. Spectra were binned to have at least 25 counts in each spectral channel, and to oversample the intrinsic energy resolution of each EPIC camera by a factor not larger than 3. Spectral fits were performed in the $0.35-15 \mathrm{keV}$ and $0.5-10 \mathrm{keV}$ energy ranges for the pn and MOS, respectively A fit with a one-component model ${ }^{1}$ is clearly inadequate (if a power-law

\footnotetext{
1 All the models discussed in this paper are photoabsorbed by cold matter, whose column density is held fixed to the contribution of our Galaxy along the line-of-sight to Mkn 668: $N_{\mathrm{H}, \mathrm{Gal}}=1.4 \times 10^{20} \mathrm{~cm}^{-2}$ (Dickey \& Lockman 1990).
} 
Table 2. Best-fit parameters and results for global fits to the nuclear spectra of Mkn 668 . Models legenda: $\# 1=B K N P O+G A$; $\# 2=$ WA ${ }^{\text {soft }} \times$ $\left(\mathrm{PO}^{\text {soft }}+2 \times \mathrm{GA}\right)+\mathrm{WA}^{\text {hard }} \times\left(\mathrm{PO}^{\text {hard }}+\mathrm{GA}\right) ; \# 3=\mathrm{WA} \times\left(\mathrm{PO}^{\text {soft }}+\mathrm{PEXRAV}+3 \times \mathrm{GA}\right) ; \# 4=\mathrm{WA} \times(\mathrm{MEKAL}+\mathrm{PEXRAV}+\mathrm{GA})$, where: $\mathrm{PO}=$ power-law; $\mathrm{BKNPO}=$ broken power-law; $\mathrm{PEXRAV}=\mathrm{Compton}-$ reflection; $\mathrm{MEKAL}=$ thermal plasma emission; $\mathrm{GA}=\mathrm{Gaussian}$ emission line; $\mathrm{WA}=$ photoelectric absorption

\begin{tabular}{lccccccc}
\hline \hline Model & $\begin{array}{c}N_{\mathrm{H}}^{\text {soft }} \\
\left(10^{21} \mathrm{~cm}^{-2}\right)\end{array}$ & $\begin{array}{c}N_{\mathrm{H}}^{\text {hard }} \\
\left(10^{23} \mathrm{~cm}^{-2}\right)\end{array}$ & $\Gamma_{\text {hard }}{ }^{a}$ & $\begin{array}{c}E_{\text {break }} \\
(\mathrm{keV})\end{array}$ & $\Gamma_{\text {soft }}$ & $\begin{array}{c}k T \\
(\mathrm{keV})\end{array}$ & $\chi^{2} / v$ \\
\hline \multicolumn{2}{l}{ XMM-Newton } & & & & & & \\
$\# 1$ & $1.3 \pm_{1.0}^{1.2}$ & $\ldots$ & $0.7 \pm_{0.4}^{0.3}$ & $2.3 \pm_{0.4}^{0.8}$ & $2.1 \pm_{0.3}^{0.6}$ & $\ldots$ & $34.0 / 41$ \\
$\# 2$ & $1.2 \pm_{0.8}^{0.9}$ & $2.4 \pm_{0.8}^{1.0}$ & $2.0 \pm_{0.3}^{0.4}$ & $\ldots$ & $\equiv \Gamma_{\text {hard }}$ & $\ldots$ & $31.1 / 41$ \\
$\# 3 \mathrm{a}^{b}$ & $1.1 \pm 0.5$ & $\ldots$ & $2.21 \pm_{0.14}^{0.19}{ }^{a}$ & $\ldots$ & $\equiv \Gamma_{\text {hard }}$ & $\ldots$ & $34.8 / 42$ \\
$\# 3 \mathrm{~b}$ & $1.9 \pm_{1.0}^{1.3}$ & $\ldots$ & $2^{a, c}$ & $\ldots$ & $2.6 \pm 0.5$ & $\ldots$ & $32.2 / 42$ \\
$\# 4$ & $0.9 \pm_{0.6}^{0.8}$ & $\ldots$ & $2.2 \pm 0.4^{a}$ & $\ldots$ & $\ldots$ & $1.3 \pm_{0.4}^{0.8}$ & $33.2 / 42$ \\
ASCA & & & & & & & \\
$\# 3 \mathrm{a}^{b}$ & $<0.8$ & $\ldots$ & $1.2 \pm_{0.3}^{2.0 a}$ & $\ldots$ & $\equiv \Gamma_{\text {hard }}$ & $\ldots$ & $79.7 / 77$ \\
\hline
\end{tabular}

${ }^{a}$ Intrinsic, for the Compton-reflection model; ${ }^{b}$ "baseline model" in text; ${ }^{c}$ fixed.

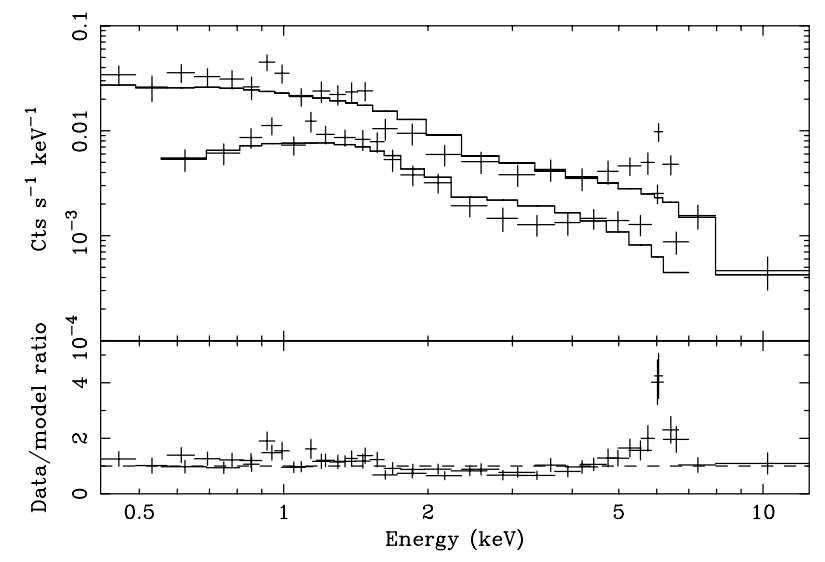

Fig. 1. Spectra (upper panel) and residuals in units of data/model ratio (lower panel) when a photoelectrically absorbed power- law model is simultaneously fit to the MOS and pn spectra of Mkn 668. Each data point corresponds to a signal-to-noise ratio larger than 3 .

model is used: $\chi^{2}=111.0 / 47 v$, where $v$ indicates the number of degrees of freedom; the residuals against this fit are shown in Fig. 1). Phenomenologically, a good fit can be obtained with a two continuum model such a broken power-law (cf. Table 1), provided that a Gaussian emission line is included to account for a narrow-band feature around $6 \mathrm{keV}$ (observer's frame). The F-test for the addition of emission line feature to the broken power-law continuum is highly significant: $\Delta \chi^{2} / \Delta v=36.5 / 3$, which corresponds to a $99.9992 \%$ confidence level according to the F-test ${ }^{2} \mathrm{~A}$ zoom of the spectral area around $6 \mathrm{keV}$ is shown in Fig. 2 (only pn data are shown for clarity), where a constant linear rebinning in the energy space has been applied to prevent weak, narrow-band features from being visually smeared. The emission feature clearly stands out against the underlying continuum. We have

\footnotetext{
${ }^{2}$ Following Protassov et al. (2002) we have applied the F-test without imposing any constraints on the Gaussian profile normalization sign.
}

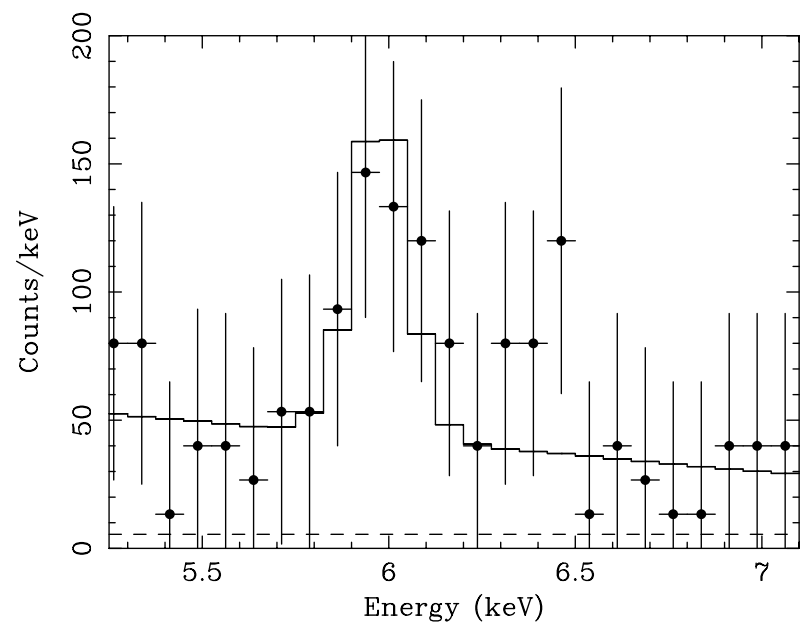

Fig. 2. pn non-background subtracted spectrum in the $5.25-7.25 \mathrm{keV}$ energy band. Data points have been rebinned with a constant width of $75 \mathrm{eV}$ (which corresponds to about one-third of the intrinsic energy resolution). The solid line represents a fit with a power-law continuum and an unresolved Gaussian profile. The background level is indicated by the dashed line.

simultaneously fitted the non background-subtracted pn and MOS spectra in the 5.25-7.25 keV energy band, using the C-statistics (which is appropriate for spectra, whose channel photon distribution is purely Poissonian). In this fit, the local pn (MOS) background has been modeled with a simple powerlaw (a good approximation in such a small energy range), with normalization $1.41 \times 10^{-6}\left(2.61 \times 10^{-7}\right) \mathrm{keV} \mathrm{cm}^{-2} \mathrm{~s}^{-1}$ and photon index $0.38(-0.52)$. The local continuum has been approximated by a power-law as well. A single narrow emission line leaves residuals bluewards the energy centroid. They can be accounted for either by a single broad line, with centroid energy $E_{\mathrm{c}}=6.44 \pm_{0.04}^{0.08} \mathrm{keV}, \sigma=100 \pm_{50}^{100} \mathrm{eV}$ and intensity $I_{\mathrm{c}}=\left(4.2 \pm_{0.8}^{1.8}\right) \times 10^{-6} \mathrm{~cm}^{-2} \mathrm{~s}^{-1}$, or by a combination of three narrow lines, whose energies are well consistent with neutral, He-like, and H-like iron. (cf. Table 3). Total net counts in the 
Table 3. Properties of the emission line components for a "local" (power-law and narrow Gaussian profiles in the 5.25-7.25 keV energy band) and a "global fit" (model \#3a in Table 2). Errors are 1- $\sigma$ for one interesting parameter. $E W \mathrm{~s}$ are calculated against the proper continuum (details in text).

\begin{tabular}{lcc}
\hline \hline & "Local fit" & "Global fit" \\
\hline$E_{\mathrm{c}}^{(1)}(\mathrm{keV})$ & $6.42 \pm_{0.02}^{0.04}$ & $6.4^{\dagger}$ \\
$I_{\mathrm{c}}^{(1)}\left(10^{-6} \mathrm{~cm}^{-2} \mathrm{~s}^{-1}\right)$ & $3.4 \pm_{1.2}^{0.9}$ & $2.7 \pm_{0.8}^{0.7}$ \\
$E W^{(1)}(\mathrm{eV})$ & $\ldots$ & 630 \\
$E_{\mathrm{c}}^{(2)}(\mathrm{keV})$ & $6.70 \pm_{0.18}^{0.12}$ & $6.7^{\dagger}$ \\
$I_{\mathrm{c}}^{(2)}\left(10^{-6} \mathrm{~cm}^{-2} \mathrm{~s}^{-1}\right)$ & $0.9 \pm_{0.7}^{0.8}$ & $2.3 \pm_{1.4}^{0.9}$ \\
$E W^{(2)}(\mathrm{eV})$ & $\ldots$ & 3400 \\
$E_{\mathrm{c}}^{(3)}(\mathrm{keV})$ & $6.97 \pm_{0.08}^{0.07}$ & $6.96^{\dagger}$ \\
$I_{\mathrm{c}}^{(3)}\left(10^{-6} \mathrm{~cm}^{-2} \mathrm{~s}^{-1}\right)$ & $1.2 \pm_{0.7}^{0.9}$ & $<1.0$ \\
$E W^{(3)}(\mathrm{eV})$ & $\ldots$ & $<1600$ \\
\hline
\end{tabular}

$\dagger$ Fixed.

line are $110 \pm 50$. Although these "local fit" results may be affected by uncertainties in the determination of the local continuum, and need therefore to be regarded with caution, they represent a pathfinder to guide the global fitting.

The line complex has a very large Equivalent Width $(E W=$ $\left.1.5 \pm_{0.4}^{0.5} \mathrm{keV}\right)$. Equivalent widths of this order can be most naturally explained if the continuum underlying the emission line is partially or totally obscured, whereas the line photons reach us through an optical path, which does not intercept the obscuring matter (Turner et al. 1997; Matt et al. 2000). In the broken power-law fit of the Mkn 668 spectra, the photon index above $\simeq 2.3 \mathrm{keV}\left(\Gamma_{\text {hard }}=0.7 \pm 0.3\right)$ is indeed flatter than typically observed in unobscured AGN (Nandra et al. 1997; Reeves \& Turner 2000), and therefore advocates for obscuration of the nuclear emission.

In light of the above results, we tested a scenario whereby the hard X-ray continuum is produced by an heavily absorbed nuclear continuum, whereas the soft excess and the $\mathrm{He}$ - and $\mathrm{H}$-like iron lines are produced by electron scattering of the same nuclear continuum. In practical terms, we have substituted the broken power-law continuum with the combination of two power-laws, photoabsorbed by column densities $N_{\mathrm{H}}^{\text {soft }}$ and $N_{\mathrm{H}}^{\text {hard }}$, respectively. Even if the soft and hard photon indices are tied together, the fit is good $\left(\chi^{2}=31.5 / 42\right)$. The flat highenergy spectrum is well represented by a "standard" AGN spectral index $\left(\Gamma_{\text {hard }}=2.0 \pm_{0.3}^{0.4}\right)$ absorbed by $N_{\mathrm{H}}^{\text {hard }}=2.4 \pm_{0.8}^{1.0} \times$ $10^{23} \mathrm{~cm}^{-2}$. In this scenario, however, the equivalent width of the neutral iron line $(E W \simeq 700 \mathrm{eV})$ remains unexplained (Leahy \& Creighton 1993). A comparatively good fit $\left(\chi^{2}=\right.$ $35.7 / 43)$ is obtained if the hard X-ray emission is modeled with a "bare" Compton-reflection component (model pexrav in XsPEC; Magdziarz \& Zdziarski 1995), therefore assuming that a Compton-thick absorber $\left(N_{\mathrm{H}} \geq \sigma_{\mathrm{t}}^{-1} \simeq 1.5 \times 10^{24} \mathrm{~cm}^{-2}\right)$, totally suppresses the nuclear emission below $10 \mathrm{keV}$ (Matt et al. 1999b). The large iron line $E W(\simeq 630 \mathrm{eV})$ is naturally produced in this scenario (Ghisellini et al. 1994; Krolik et al. 1994). Any transmitted component is constrained to be absorbed by $N_{\mathrm{H}}^{\text {hard }} \gtrsim 9 \times 10^{23} \mathrm{~cm}^{-2}$ (for $\Gamma=2.21$ and a warm scattering fraction of 5\%). In Table 3 we summarize the properties of the emission line complex in the "global fit" model, if one assumes a 3-components (neutral, He-like and H-like iron) decomposition. The $E W$ are calculated with respect to the continuum against which they are supposedly produced: the Compton-reflection continuum for the neutral component, the scattered continuum for the ionized components. The normalizations of the continuum components in this model are: $N^{\text {reflection }}=(1.6 \pm 0.5) \times 10^{-3}$, and $N^{\text {scattering }}=\left(3.5 \pm_{0.4}^{0.5}\right) \times$ $10^{-5} \mathrm{keV} \mathrm{cm}^{-2} \mathrm{~s}^{-1}$, for the Compton-reflection and the scattering component, respectively. They corresponds to observed fluxes of $\left(0.6 \pm_{0.2}^{0.6}\right)$ and $\left(3.1 \pm_{0.7}^{1.0}\right) \times 10^{-13} \mathrm{erg} \mathrm{cm}^{-1} \mathrm{~s}^{-2}$ in the $0.5-2 \mathrm{keV}$ and $2-10 \mathrm{keV}$ energy bands, respectively, and to unabsorbed luminosities of $\left(0.8 \pm_{0.3}^{0.8}\right)$ and $\left(4.1 \pm_{0.9}^{1.3}\right) \times 10^{42} \mathrm{~cm}^{-2}$ in the same energy bands. We will refer to this model (\#3a in Table 1) as "baseline model" hereinafter.

An alternative origin for the ionized components of the emission line complex is thermal emission in an optically thin, collisionally ionized plasma (Boller et al. 2003). We have therefore tried to fit the spectrum with a combination of a Comptonreflection continuum, a single narrow FeI emission line, and thermal emission component, using for the last the mekal implementation in XsPEC (Mewe et al. 1985). The fit is again statistically acceptable. However, the temperature of the thermal component $\left(k T \simeq 1.3 \mathrm{keV}\right.$, with $\left.Z<0.14 Z_{\odot}\right)$ is not high enough to contribute significantly to the ionized lines emission in the iron regime. The addition of a further thermal component is not required from the statistical point of view. It is also worth mentioning that a model constituted by two thermal components does not fit well the EPIC spectra $\left(\chi^{2} / v=65.2 / 42\right)$.

\section{Comparison with previous X-ray observations}

\subsection{ASCA observations}

Mkn 668 was observed by ASCA between January 4 and January 6, 1999. Preliminary results on this observation are discussed by Guainazzi et al. (2003), who stress that, due to the low statistics, it is impossible to obtain a fully unambiguous spectral deconvolution. We extracted spectra from the screened event lists available at the HEASARC ASCA archive, employing circular regions of $3^{\prime}$ radius around the source centroid, after retaining only SIS events with standard $(0,2$, $3,4)$ grades. Total exposure time was $65.5 \mathrm{ks}(72.1 \mathrm{ks})$ for the SIS (GIS) cameras. Background spectra were extracted from field-of-view regions, free from contaminating sources. Response matrices appropriate for the extracted spectra were generated with LHEASOFT v5.1. Applying the baseline model simultaneously to the spectra of all the ASCA instruments one gets an acceptable fit $\left(\chi^{2} / v=79.7 / 77\right)$. Both the continuum and the line best-fit parameters are consistent with those derived from the XMM-Newton observation, within the admittedly large statistical uncertainties. In particular, a large $E W$ iron line is marginally detected in the ASCA observation as well, suggesting, alongside with the flat hard X-ray continuum, a Compton-reflection dominated spectrum. The parameters of the iron line complex, when fit with a single 
Table 4. Summary of spectral results for the ROSAT observations of Mkn 668 (details in text). The superscript refers to HRI ("H") or PSPC ("P") observations.

\begin{tabular}{lccc}
\hline \hline Date & $\begin{array}{c}N_{\mathrm{H}} \\
\left(10^{21} \mathrm{~cm}^{-2}\right)\end{array}$ & $\Gamma$ & $\begin{array}{c}0.1-2.4 \mathrm{keV} \\
\text { rate }\left(10^{-3} \mathrm{~s}^{-1}\right)\end{array}$ \\
\hline $9 / 1 / 92^{\mathrm{H}}$ & $\ldots$ & $\ldots$ & $<2.4$ \\
$23 / 1 / 96^{\mathrm{H}}$ & $\ldots$ & $\ldots$ & $2.0 \pm 0.4$ \\
$10 / 7 / 96^{\mathrm{P}}$ & $0.8 \pm_{0.5}^{3.1}$ & $2.4 \pm_{1.1}^{2.1}$ & $7.9 \pm 1.3$ \\
$12 / 1 / 1998^{\mathrm{H}}$ & $\ldots$ & $\ldots$ & $1.6 \pm 0.3$ \\
\hline
\end{tabular}

Gaussian profile, are: $E_{\mathrm{c}}=6.7 \pm_{0.2}^{0.4} \mathrm{keV}, \sigma=250 \pm_{250}^{350} \mathrm{eV}, I_{\mathrm{c}}=$ $\left(7 \pm_{4}^{8}\right) \times 10^{-6} \mathrm{~cm}^{-2} \mathrm{~s}^{-1}$ (corresponding to an $E W=850 \mathrm{eV}$ ). The $2-10 \mathrm{keV}$ flux observed by ASCA is almost double as measured during the XMM-Newton observation: $\left(6.1 \pm_{1.2}^{1.3}\right) \times$ $10^{-13} \mathrm{erg} \mathrm{cm}^{-2} \mathrm{~s}^{-1}$. This is suggestive of an historical change of the nuclear emission output power by a factor of 2 between the two observations, although a contribution from a larger brightening of one of the sources detected by XMM-Newton and encompassed in the large ASCA aperture cannot be ruled out. On the other hand, the $0.5-2 \mathrm{keV}$ ASCA flux is consistent with the later XMM-Newton measurement: $\left(0.9 \pm_{0.3}^{0.4}\right) \times$ $10^{-13} \mathrm{erg} \mathrm{cm}^{-2} \mathrm{~s}^{-1}$.

An earlier (July 17 1998) and shorter (GIS exposure time $33 \mathrm{ks})$ ASCA observation produced a $6 \sigma$ detection, corresponding to a total $0.5-10 \mathrm{keV}$ unabsorbed flux of $(5 \pm 2) \times$ $10^{-13} \mathrm{erg} \mathrm{cm}^{-2} \mathrm{~s}^{-1}$. No useful spectral information can be derived from this observation.

\subsection{ROSAT observations}

Mkn 668 was observed 4 times by ROSAT, one of them with the Position Sensitive Proportional Counter (PSPC) detector, and the remaining with the High Resolution Imager (HRI). Preliminary results of the former observation are discussed by Zhang \& Marscher (1994). We have reanalyzed the ROSAT public data, extracting calibrated event lists from the public archive. The results of the analysis are summarized in Table 4. The PSPC spectrum can be adequately fit in the $0.1-2.4 \mathrm{keV}$ energy band by an absorbed power-law $\left(\chi^{2} / v=17.6 / 20\right)$. In Fig. 3 we compile eleven years of Mkn 668 soft X-ray flux measurements. The HRI fluxes have been extrapolated from the observed count rate, assuming an absorbed powerlaw with $N_{\mathrm{H}}=1.4 \times 10^{21} \mathrm{~cm}^{-2}$ and $\Gamma=2.2$ (best-fit of the XMM-Newton EPIC spectra in the $0.5-2 \mathrm{keV}$ energy band alone). Caution must be applied in interpreting this plot, due to the uncertainties induced by the unknown spectral shape in the ROSAT/HRI measurements, and to the potential contamination of serendipitous sources in the large ASCA aperture. Nonetheless, the observed soft X-ray variability dynamical range in Mkn 668 is less than a factor 50\%. The XMM-Newton observation has possibly caught one of its faintest soft X-ray states ever.

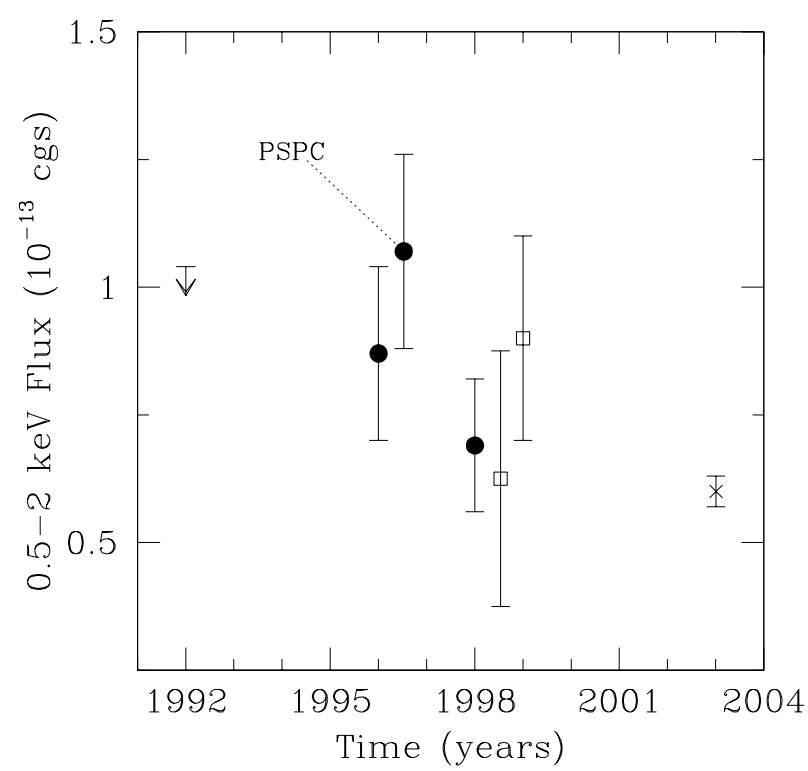

Fig. 3. Historical $0.5-2 \mathrm{keV}$ flux light curve of Mkn 668. Measurements are corrected for absorption. Error bars represent the propagation of the 1- $\sigma$ uncertainties on the count rates. Filled circles and upper limit: ROSAT; empty squares: ASCA; cross: XMM-Newton.

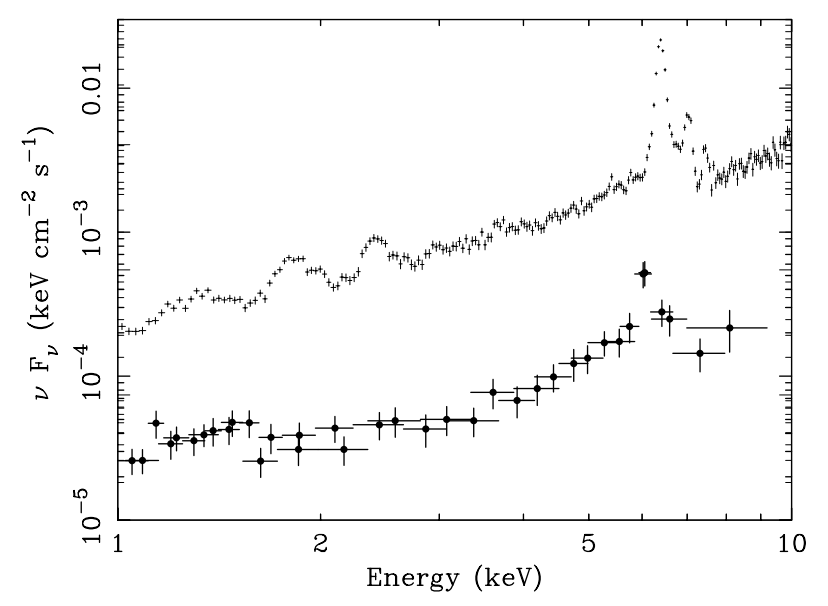

Fig. 4. X-ray SEDs for the Circinus Galaxy ( $z=0.0015$; top, pn data) and the Mkn 668 ( $z=0.077$; bottom, EPIC data) nuclei.

\section{Discussion}

\subsection{Mkn 668: A Compton-thick Seyfert 1 galaxy}

The X-ray spectrum of Mkn 668 exhibits two distinct components. At energies $E \gtrsim 2.5 \mathrm{keV}$ the spectrum is remarkably flat. This evidence, alongside with the prominent $\mathrm{K}_{\alpha}$ fluorescent emission line, represents a clear indication for a Comptonreflection dominated spectrum (Matt et al. 2000, and references therein). The direct view of the primary nuclear emission is hindered by photoelectric absorption with a column density $N_{\mathrm{H}} \gtrsim 9 \times 10^{23} \mathrm{~cm}^{-2}$. This is confirmed by the overall similarity between the X-ray Spectral Energy Distributions (SEDs) of Mkn 668 and of the Circinus Galaxy (cf. Fig. 4). The latter is the closest Compton-thick AGN known; its flat hard X-ray continuum and prominent line emission complex are interpreted in terms of Compton-reflection of an otherwise invisible (below 
$10 \mathrm{keV}$ ) nuclear emission (Matt et al. 1999a; Molendi et al. 2003).

If there is little doubt that the X-ray appearance of Mkn 668 is that of a highly obscured AGN, its optical spectrum shows features more typical of a Broad Line Radio Galaxy (Blake et al. 1970).

Marziani et al. (1993) discuss the peculiar properties of the broad optical lines in Mkn 668. The peaks of the broad $\mathrm{H}_{\alpha}$ and $\mathrm{H}_{\beta}$ components are displaced to the red with respect to the centroid of the narrow components of the same lines. The observed correlation between line luminosity and centroid displacement is well explained by radiative acceleration in a system of outflowing clouds. Marziani et al. (1993) therefore conclude that broad line emission in this galaxy partly occurs in a cone of half-opening angle $\simeq 12^{\circ}$ seen at inclination angles $l \lesssim 30^{\circ}$.

To our knowledge, Mkn 668 is the first known nontransient Seyfert 1 galaxy, whose X-ray spectrum is Comptonreflection dominated, hence suggesting that the nucleus is covered by a Compton-thick absorber. The only analogous case reported in the literature is the "off-state" of NGC 4051 (Guainazzi et al. 1998). The X-ray spectrum of this Narrow Line Seyfert 1 Galaxy was discovered by BeppoSAX to be Compton-reflection dominated during $\mathrm{a} \simeq 100$ days-long period when the total X-ray output decreased by a factor of $\simeq 10^{2}$ with respect to its standard activity level (Uttley et al. 1999).

However, no evidence exists in Mkn 668 for large variations of the AGN X-ray output. In hard X-rays, only the measurements described in this paper are available, which limit the variability dynamical range above $2 \mathrm{keV}$ within a modest factor of 2 . In the soft X-ray range, where a better temporal coverage is available, there is no evidence for a variability dynamical range larger than $50 \%$ (cf. Fig. 3). There is therefore no direct evidence so far that extreme X-ray variability is the culprit for the discrepancy between the optical classification and the X-ray spectral properties in Mkn 668.

\subsection{The soft $X$-ray spectrum}

The soft X-ray spectrum of Mkn 668 is dominated by a steeper component, whose origin cannot be unambiguously determined on statistical basis alone. No clear evidence for soft X-ray emission lines is found. The $90 \%$ upper limits on the equivalent widths of mid-Z He-like $\mathrm{K}_{\alpha}$ fluorescent lines (i.e.: NE IX; MG XI; SI XIII; SXV) are in range 40-80 eV (against the scattering continuum), therefore a factor 3 to 10 lower than typically observed in "warm scattered" Seyfert 2 galaxies (Guainazzi et al. 1999). On the other hand, the profile of the emission complex in the iron fluorescent regime, significantly more complex than a single unresolved component, suggests a contribution from highly ionized iron species. The $E W$ of the iron He-like emission line $\left(E W=3.4 \pm_{1.3}^{2.0} \mathrm{keV}\right)$ is consistent with optically thin resonant scattering (Matt et al. 1996) by plasma with standard solar abundances. A substantial contribution to the ionized iron line from thermal plasma is unlikely.

Although the warm scattering scenario nicely explains simultaneously the soft X-ray continuum and the FeXXV fluorescent emission, the agreement between the spectral energy indices in the radio $\left(\alpha_{\text {radio }} \simeq-1.1\right)$ and in the soft X-ray $\left(\alpha_{\mathrm{X}} \simeq-1.2\right)$ may indicate that at least part of the soft X-ray photons are produced by non-thermal processes, occurring in the same electron population responsible for the emission in the radio band. However, if we make use of Kameno et al. (2000) best-fit synchrotron model for the radio spectrum of the NE lobe, assuming free-free absorption with $\tau_{\mathrm{ff}}=6.4 \pm$ 0.4 , the extrapolation underpredicts the soft X-ray counts by at least 0.5 dex (the discrepancy in the hard X-ray band is obviously larger). A monitoring of the $4.9 \mathrm{GHz}$ flux in Mkn 668 between 1975 and 1995 detected only a 20\% gradual decrease of the radio flux (Stanghellini et al. 1997), ruling out an explanation of the discrepancy in terms of lobe radio flux variability. There is actually evidence for a steepening of the lobe spectrum with increasing frequency (Dallacasa et al. 2000), with $\alpha_{\text {radio }}$ decreasing from $\simeq-0.7$ in the $8.4-15 \mathrm{GHz}$ to $\simeq-1.4$ in the $15-22 \mathrm{GHz}$ frequency range. Finally, the radio spectra available so far do not resolve the hotspot, and represent therefore a blending between the hotspot and the surrounding lobe. Although only strictly simultaneous and aperture-matched observations of Mkn 668 in the radio and X-ray bands will be able to definitely rule out this possibility, there is no clear evidence so far that synchrotron emission by an electron population consistent with the $1-15 \mathrm{GHz}$ spectrum represents a major contribution to the soft X-ray spectrum.

Alternatively, Inverse Compton (IC) effects have been suggested to be the dominant process in large-scale X-ray jets (Tavecchio et al. 2000; Celotti et al. 2001), particularly in GPS quasars (Siemiginowska et al. 2002, 2003). The seed photons scattered off the lobe electrons may be the same synchrotron photons responsible for the radio hotspot emission (Synchrotron Self-Compton, SSC, model), Cosmic Microwave Background photons (CMB), or could be locally produced in the AGN environment. In the SSC scenario, the ratio between the IC and the synchrotron luminosity is given by the ratio between the photon $\left(u_{\mathrm{e}}\right)$ and the magnetic energy density $\left(u_{\mathrm{m}}\right)$, which in turn can be expressed as (Tavecchio et al. 1998):

$\frac{u_{\mathrm{e}}}{u_{\mathrm{m}}} \sim 6 \times 10^{-4} v_{8.4} T_{11}^{5}$

where $T_{11}$ is the brightness temperature at the frequency $v_{8.4}$, in units of $8.4 \mathrm{GHz}$. At this frequency, $T_{11}=0.5$ for Mkn 668 (Ghisellini et al. 1993). It is therefore implausible that this process significantly contributes to the soft X-rays.

The energy density of the CMB at Mkn 668 redshift is $\sim 5 \times$ $10^{-13} \gamma \mathrm{erg} \mathrm{cm}^{-3}$, where $\gamma$ is the electron Lorentz factor. One may estimate the magnetic field in the hotspot region $B_{\mathrm{IC}}$ applying the Harris \& Krawczynski (2002) prescription to the observed ratio between the radio $\left(S_{5 \mathrm{GHz}} \simeq 2.7 \mathrm{Jy}\right.$; Stanghellini et al. 1996) and the soft X-ray $\left(L_{X}=8 \times 10^{41} \mathrm{erg} \mathrm{s}^{-1}\right)$ luminosity. Assuming an inclination angle of $15^{\circ}\left(45^{\circ}\right)^{3}$, one gets $B_{\mathrm{IC}} \sim 3 \mu \mathrm{G}(5 \mu \mathrm{G})$. This is far too low with respect to the minimum energy condition magnetic field ( $B_{\mathrm{me}}$; Pacholcyzk 1970). The observed $5 \mathrm{GHz}$ integrated flux density in Mkn 668, implies $B_{\mathrm{me}} \sim 60 \theta_{\mathrm{sec}}^{-4 / 7}(\sin l)^{-3 / 7} \mu \mathrm{G}$ (Miley 1980), where $\theta_{\mathrm{sec}}$ is

${ }^{3}$ Stanghellini et al. (1997) estimate $\imath \simeq 45^{\circ}$ on the basis of the Mkn 668 optical properties, and assuming that the radio axis is perpendicular to the plane of the galaxy. 
the hotspot size in arcseconds. In Mkn $668 \theta_{\mathrm{sec}} \simeq 0.7 \times 0.2$ mas (Barone 2003).

The local AGN radiation energy density, $u_{\mathrm{AGN}}$ potentially available for Compton scattering should be dominated by the remarkably large Far Infrared Emission $\left(L_{\mathrm{FIR}} \simeq 1.4 \times 10^{11} L_{\odot}\right.$; Mazzarella et al. 1991). An exact estimate of the energy density at the radio hotspots would require a detailed knowledge of the geometry of the circumnuclear region, which is largely unknown. If the FIR-emitting dust is located within the radio structure (see Sect. 4.3.1), and the radio hotspots have a unity filling factor we estimate $u_{\mathrm{AGN}} \sim 6 \times 10^{-12}\left(\Omega_{\text {dust }} / 2 \pi\right) \mathrm{erg} \mathrm{cm}^{-3}$, where $\Omega_{\text {dust }}$ is the solid angle subtended by the radio hotspots to the dust. For moderate electron Lorentz factors, the local AGN radiation energy density can indeed exceed the CMB energy density at the hotspots, if dust and radio lobes cover regions of comparable size and/or are close.

\subsection{The nature of the X-ray absorbers in Mkn 668}

The spectral analysis of the XMM-Newton observation of the Mkn 668 reveals the presence of two different absorbing systems. Together with the Compton thick absorber covering the direct line-of-sight to the nucleus, an additional absorbing system with $N_{\mathrm{H}} \sim 10^{21} \mathrm{~cm}^{-2}$ covering the soft X-ray spectrum is required by all models presented in Sect. 2 . In this section we compare the X-ray and radio results, and derive some physical properties of the absorbing systems.

\subsubsection{The Compton-thick absorber}

We first ask whether the Compton-thick matter could be responsible for the free-free absorption of the radio hotspots (Kameno et al. 2000), and could therefore be located at a distance from the active nucleus at least larger than the halfseparation between the two lobes $(\simeq 5 \mathrm{pc})$. An estimate of the AGN intrinsic power in Mkn 668 can be inferred from the $\mathrm{O}[\mathrm{III}]$ flux $\left(5 \times 10^{-14} \mathrm{erg} \mathrm{\textrm {cm } ^ { - 2 }} \mathrm{s}^{-1}\right.$; Marziani et al. 1993). According to the correlation between the $\mathrm{O}[\mathrm{III}]$ and the $2-10 \mathrm{keV}$ luminosity $\left(\log \left(L_{2-10 \mathrm{keV}} / L_{\mathrm{O}[\mathrm{III}]}\right) \sim 1.8\right.$; Mulchaey et al. 1994; Maiolino et al. 1998), the intrinsic 2-10 keV AGN luminosity should be $\sim 9 \times 10^{43} \mathrm{erg} \mathrm{s}^{-1}$. If the size of the scattering medium is of the same order of its distance from the active nucleus, one has:

$l_{\mathrm{sc}}=\frac{\sigma_{\mathrm{T}} L_{\mathrm{int}}^{2}}{\xi L_{\mathrm{sc}}}\left(\frac{\Omega}{4 \pi}\right)$

where $l_{\mathrm{sc}}$ is the size of the scattering plasma, $L_{\mathrm{sc}}$ is the $o b$ served $2-10 \mathrm{keV}$ luminosity of the scattering component $(2 \times$ $\left.10^{42} \mathrm{erg} \mathrm{s}^{-1}\right), n$ is the scatterer particle density, $\xi \equiv L_{\mathrm{int}} / l_{\mathrm{sc}}^{2} n$ is the ionization parameter, and $\Omega /(4 \pi)$ is the solid angle subtended by the scattering matter to the nucleus. If we express: $\xi \equiv 500 \xi_{500}$ (the detection of He-like iron fluorescent emission line associated with the scattering continuum implies a ionization parameter of at least a few hundreds); and $\Omega /(4 \pi) \equiv$ $0.01[\Omega /(4 \pi)]_{0.01}$ (corresponding to a cone with a half-opening angle of $\left.15^{\circ}\right), l_{\mathrm{sc}} \sim \xi_{500}^{-1}[\Omega /(4 \pi)]_{0.01} 0.02 \mathrm{pc}$. This would be too a small size for the scattered continuum to be visible beyond an homogeneous distribution of Compton-thick matter covering the radio hotspots and the nucleus. This discrepancy is not solved even if $\Omega /(4 \pi)=0.25$ is assumed, believed to be a reasonable value in the archetypical Compton-thick Seyfert 2 galaxy NGC 1068 (Evans et al. 1991). We conclude therefore that the Compton-thick matter must be located within the separation between the radio hotspots, in agreement to typical estimates of the torus size in Seyfert galaxies (Greenhill et al. 1996, 1997; Guainazzi et al. 2000; Bianchi et al. 2001).

From its FIR luminosity, Knapp et al. (1990) estimate that $\sim 5 \times 10^{8} M_{\odot}$ of dust must be present in the core of Mkn 668 . If this dust is in the form of a torus within 10 parsecs from the core, the torus column density must exceed $3 \times 10^{25} \mathrm{~cm}^{-2}$ (in agreement with the X-ray results). The physical conditions in the torus can be investigated, using the stability conditions for X-ray illuminated torii discussed by Neufeld et al. (1994) and Maloney (1996). The condition for a torus to be fully molecular if that $\log \left(\xi_{\text {eff }}\right)<-3.4$, where $\xi_{\text {eff }}$ is the "effective" ionization parameter at the outer side of the torus, taking into account self-shielding of the ionizing continuum. Following Maloney (1996), $\xi_{\text {eff }}$ can be expressed as:

$\xi_{\mathrm{eff}}=0.17 L_{44} N_{24}^{-0.9} n_{6}^{-1} r_{\mathrm{pc}}^{-2}$

$L_{44}$ is the X-ray luminosity in units of $10^{44} \mathrm{erg} \mathrm{s}^{-1}, N_{24}$ is the column density in units of $10^{24} \mathrm{~cm}^{-2}, n_{6}$ is the particle density in units of $10^{6} \mathrm{~cm}^{-3}$, and $r_{\mathrm{pc}}$ is the distance between the torus and the active nucleus in parsecs. Moreover, a torus is in steady state if its pressure exceeds the radiation pressure. If only the gas pressure is relevant:

$n_{6} r_{\mathrm{pc}}^{2}>10^{2} L_{44} T_{3}^{-1}$

where $T_{3}$ is the average torus temperature $(600 \mathrm{~K}$ for a molecular torus: Maloney 1996). From Eq. (4): $\log n_{6}+2 \log r_{\mathrm{pc}}>$ 4.15, which implies $\log \xi_{\text {eff }}<-4.75$, in agreement with the molecular hypothesis.

\subsubsection{The Compton-thin absorber}

If the jet is opening its way through the ambient interstellar medium, each radio lobe is surrounded by a bow shock, whose precursor highly ionized clouds may give rise to freefree absorption of the radio emission. Philström et al. (2003) estimate from ram pressure arguments a particle density of $\simeq 30 \mathrm{~cm}^{-3}$ at the external surface of the bow shock generated by the "drilling jet" (Bicknell et al. 1997). If the absorbing matter is distributed in a shell surrounding the radio hotspots, its radial thickness $\Delta r_{\mathrm{ab}}$ is

$\Delta r_{\mathrm{ab}}=\frac{N_{\mathrm{H}}}{n_{\mathrm{H}}} \simeq 10 \mathrm{pc}$.

This implies that the radio and X-ray (Compton-thin) absorbing media could coincide in a layer preceding the "drilling" young radio jet. Such a medium would not contribute significantly to the X-ray emission, even if heated up to X-ray emitting temperatures. The expected $0.1-10 \mathrm{keV}$ isotropic luminosity of a $1 \mathrm{keV}$ plasma in such a geometry would be at most $\sim 2 \times$ $10^{37} \mathrm{erg} \mathrm{s}^{-1}$.

The measurement of hotspot recession velocities suggests that Mkn 668 hosts a jet still in the infancy of its development 


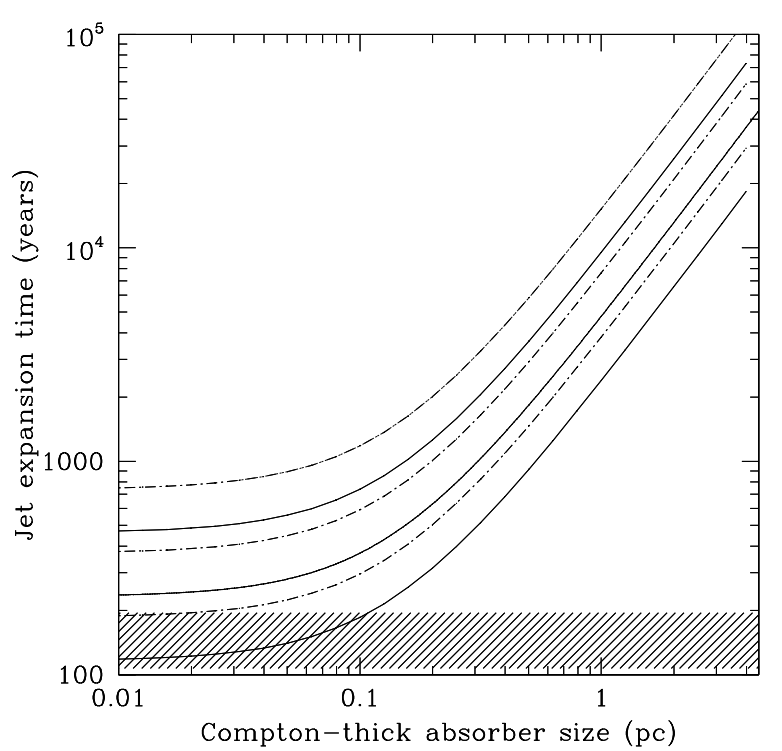

Fig. 5. Jet expansion time as a function of the physical thickness of the Compton-thick absorber. Solid lines correspond to $\imath=15^{\circ}$, dashed-dotted lines to $\imath=45^{\circ}$. From top to bottom, curves plotted in the same style correspond to values of the jet opening angle of $20^{\circ}$, $10^{\circ}$, and $5^{\circ}$, respectively. The light shaded area corresponds to the dynamical age estimate from the hotspot expansion velocity.

(Stanghellini et al. 2002; Lister 2003). Interestingly enough, however, the post-shock particle density exceeds the average value $\left(n \sim 1 \mathrm{~cm}^{-3}\right)$, sufficient to ensure the confinement of the radio jet on scales $\lesssim 1 \mathrm{kpc}$ (De Young 1993). The ultimate fate of the jet in Mkn 668 may therefore not have been written yet.

In the light of the above results, we can revise the issue of the parsec-scale radio activity age in Mkn 668. Carvalho (1998) demonstrated that the presence of a dense medium in the core of the host galaxy can significantly delay the propagation of the jet, leading to grossly inaccurate estimates of the evolution time scales. If the jet propagates under ram pressure equilibrium through a homogeneous external medium, its expansion velocity $v_{\mathrm{j}}$ can de derived from (Carvalho 1998)

$m_{\mathrm{H}} n_{\mathrm{e}} v_{\mathrm{j}}^{2} \simeq \frac{1}{3} u$

where $n_{\mathrm{e}}$ is the electron density and $u$ the jet total internal energy density. The expansion time to a distance $R_{0}$ can be therefore expressed as (Scheuer 1974; Carvalho 1985):

$t_{\mathrm{e}} \sim 4 \times 10^{5} R_{0}^{1.5}(\cos \imath)^{-1.5} L_{\text {inj }}^{-0.5} \Omega^{0.5} N_{24}^{0.5} \mathrm{~s}$

where $L_{\text {inj }}$ in the luminosity injected in the jet, and $\Omega$ is the solid angle subtended by the jet at $R_{0}$. We assume for Mkn 668 $R_{0}=5 \mathrm{pc}$, and $L_{\text {inj }} \simeq L_{\text {kin }} \sim 2 \times 10^{44} \mathrm{erg} \mathrm{s}^{-1}$ (Celotti et al. 1997). In Fig. 5 we show $t_{\mathrm{e}}$ as a function of the physical size of the Compton-thick absorber, in a simple radial geometry where a fraction $f_{\text {thick }} R_{0}$ of the optical path along the jet is (actually was) occupied by homogeneously distributed matter with number density corresponding to a total column density $5 \times$ $10^{25} \mathrm{~cm}^{-2}$, and the remaining fraction $\left(1-f_{\text {thick }}\right) R_{0}$ by matter corresponding to a total column density $10^{21} \mathrm{~cm}^{-2}$. Large inclination angles, or large thickness of the Compton-thick absorber even for moderate inclinations can significantly brake the jet, leading to an underestimate of its evolution time by one-two orders of magnitudes.

\subsection{Are the AGN in GPS galaxies obscured?}

In the left panel of Fig. 6 we show the $2-10 \mathrm{keV}$ luminosity versus the $5 \mathrm{GHz}$ luminosity for the radio-galaxy sample of Sambruna et al. (1999) and for the GPS galaxies, for which hard X-ray measurements are available so $\mathrm{far}^{4}$. The GPS galaxies - which span a range of 5 decades in both radio and X-ray luminosity - do not show any deviations from the behavior of "standard" radio galaxies. On the other hand, Fig. 6 shows the dependence of the X-ray column density on redshift ${ }^{5} .3$ out of 4 GPS galaxies exhibit an absorbing column density larger than $10^{22} \mathrm{~cm}^{-2}$, whereas only $\simeq 30 \%$ the Sambruna et al. radio galaxies do. Drawing firm conclusions on this issue from the currently available data is still impossible. However, they are at least consistent with GPS galaxies hosting AGN covered on the average by larger X-ray obscuration than large-scale radio galaxies. The ongoing XMM-Newton program will allow us to enlarge the sample of GPS galaxies with high-sensitivity hard $\mathrm{X}$-ray spectroscopic measurements, and may therefore provide the final answer to this unsettled issue.

\section{Conclusions}

Despite its optical appearance as a Seyfert 1 galaxy, the X-ray spectrum of the GPS galaxy Mkn 668 exhibits typical features of obscured AGN:

- Above $\simeq 2.5 \mathrm{keV}$, the spectrum is flat and characterized by a prominent $(E W \simeq 600 \mathrm{eV})$ neutral iron $\mathrm{K}_{\alpha}$ emission line. This suggests that Compton-reflection dominates in this energy range, alongside with fluorescence above a heavily absorbed primary nuclear continuum.

- The column density of the absorber covering the primary emission is constrained to be larger than $9 \times 10^{23} \mathrm{~cm}^{-2}$; however values larger than several $10^{25} \mathrm{~cm}^{-2}$ are more likely if the absorber reprocesses the AGN emission into the remarkably intense observed FIR luminosity.

- The bulk of the soft X-rays is due to a steeper $(\Gamma \simeq 2.0-2.5)$ power-law, absorbed by a further column density $N_{\mathrm{H}}^{\text {soft }} \sim$ $10^{21} \mathrm{~cm}^{-2}$. We associate this component with the observed fluorescent $\mathrm{K}_{\alpha}$ emission from He-like iron.

- (Warm)-scattering of the nuclear radiation is the most straightforward explanation for the soft $\mathrm{X}$-ray emission and the Fexxv fluorescent $\mathrm{K}_{\alpha}$ iron line. Nonetheless, a contribution to soft X-rays from IR photons up-scattered by the hotspot electron population cannot be ruled out, although a quantitative estimate would require a detailed knowledge of the galaxy core geometry.

- We locate the Compton-thick absorber in the innermost region of the galaxy core, within the radio hotspots.

${ }^{4}$ NGC 1052, Guainazzi et al. (2000); 1345+125, O'Dea et al. (2000); Mkn 668, this paper; Q2127+040. Siemiginowska et al., in preparation.

5 Whenever more than one absorbing system is detected, the plot in Fig. 6 displays that with the largest column density. 

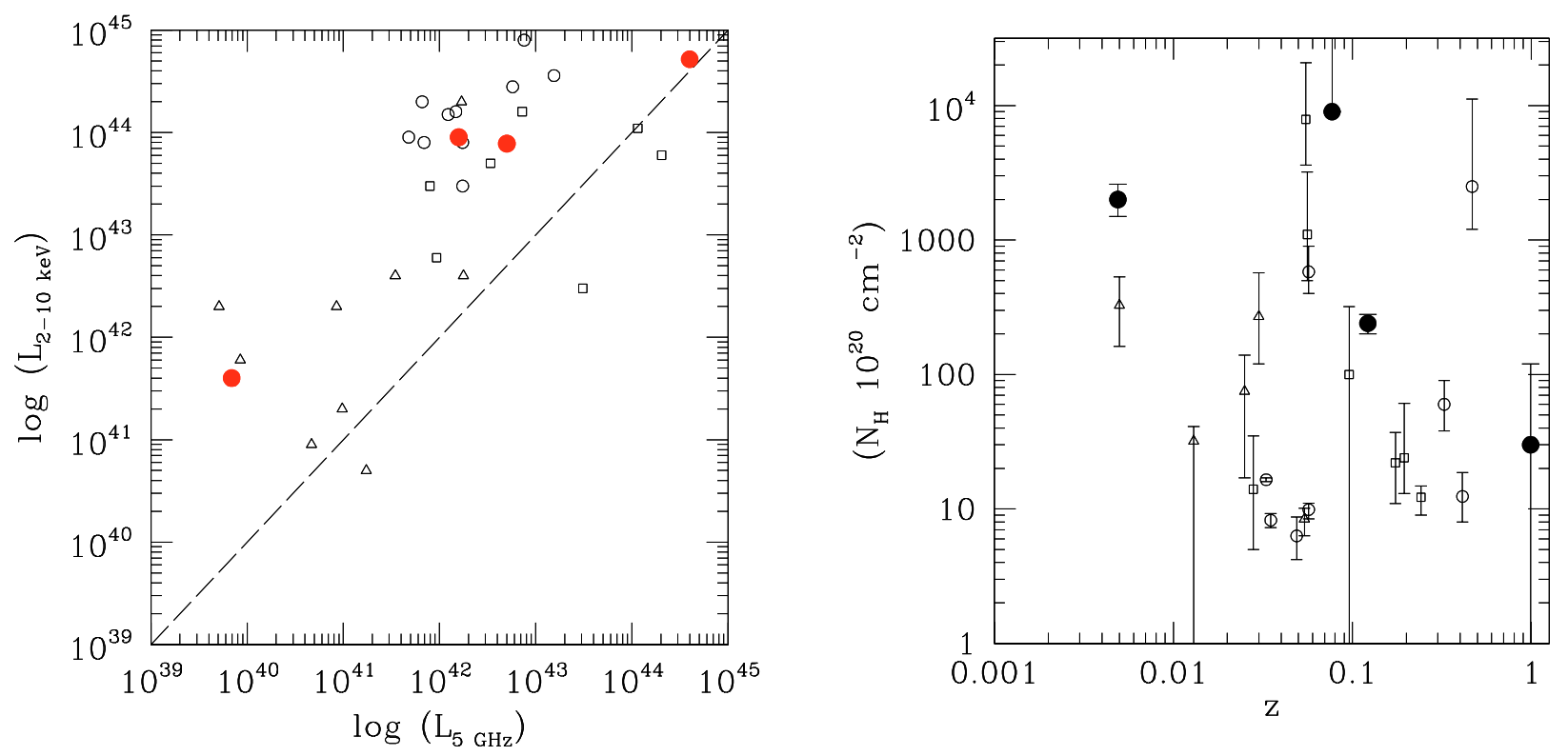

Fig. 6. Left panel: $5 \mathrm{GHz}$ versus $2-10 \mathrm{keV}$ luminosity for the radio galaxies of the Sambruna et al. (1999) ASCA sample, and the GPS galaxies for which hard X-ray measurements are available. Empty circles: broad-line radio galaxies; empty squares: narrow-line radio galaxies; empty triangles: radio galaxies; filled circles: GPS galaxies. Right panel: $N_{\mathrm{H}}$ versus redshift distribution for the same sample.

In order to be visible, the scattering plasma needs to be located beyond the visible outer rim of the Compton-thick absorbing system (what is commonly observed in Seyfert 2 galaxies), probably colocated or at least along the same line-of-sight as the unobscured BLR (what is instead not usual in Seyfert 2 galaxies, where BLRs are hidden from out direct view).

- We identify the X-ray Compton-thin absorber with matter responsible for free-free absorption of the radio source. In this scenario, this matter is probably distributed in $\mathrm{a} \simeq 10 \mathrm{pc}$ shell of relatively dense $\left(n \simeq 30 \mathrm{~cm}^{-3}\right.$ ) matter compressed by the jet bow shock.

The discrepancy between the optical classification and the $\mathrm{X}$-ray spectral properties in this object does not fit the 0-th order unification scenario for AGN (Antonucci 1993; Barthel 1989). This might be due to an uncorrelated variability between the two bands, driven by large amplitude variation in the overall energy output of the AGN, as already observed in NGC 4051 (Guainazzi et al. 1998), or in the so-called “changing-look" Seyfert 2 galaxies (Matt et al. 2003b, and references therein). However, there is no evidence for large variation of the AGN power in Mkn 668. Another possibility is that the BLRs are seen in reflected, rather than in direct, light (see, e.g., the discussion in Matt et al. 2003a). Given the large $E W$ of the broad optical lines (Marziani et al. 1993), this would imply a large covering factor for the reflector. However, the discrepancy may be otherwise related to the peculiar properties of this class of radio object. "Young" AGN, triggered by recent merging episodes, may have more compact torii, which do not effectively cover the direct view of the broad line region. Alternatively, we may be observing the AGN through the lineof-sight recently opened by the "drilling" jet, which frees the view of the broad line regions. In this case, a geometry such as a warped disk may still ensure simultaneous obscuration of the
AGN. It is in principle possible as well that Mkn 668 hosts an "intrinsic" type 2 AGN, and that the BLRs are produced by the interaction of gas with the expanding radio lobes. Bicknell et al. (1997) make specific predictions on the luminosity of [OIII] and $\mathrm{H}_{\alpha}+[\mathrm{NII}]$ optical lines produced in this scenario. The observed luminosities in Mkn 668 ( a few $10^{40} \mathrm{erg} \mathrm{s}^{-1}$; Marziani et al. 1993) are in good agreement with their model for reasonable values of the ratio between the lobe energy and the radio power (1 Jy at $1.7 \mathrm{GHz}$; Stanghellini et al. 1997). However, the optical lines are probably too broad to be produced by such a mechanism. The upper limit on the velocity of clouds accelerated by a bow shock can be estimated by imposing that the shocked gas cooling time is lower than the radio source dynamical age (O'Dea et al. 2002):

$v_{\mathrm{cl}} \lesssim 50 R_{\mathrm{pc}}^{1 / 3} n_{\mathrm{ps}, 100}^{1 / 3} v_{\mathrm{bs}}^{-1 / 3} \leq 60 \mathrm{~km} \mathrm{~s}^{-1}$

where $R_{\mathrm{pc}}$ is the distance (in parsecs) from the nucleus to the bow shock, $n_{\mathrm{ps}, 100}$ is the post-shock density in units of $100 \mathrm{~cm}^{-3}$, and $v_{\mathrm{bs}}$ is the bow shock velocity in units of the speed of light $(\simeq 0.1$ for Mkn 668; Stanghellini et al. 2002). On the other hand, the observed $\mathrm{H}_{\alpha} F W H M$ is $\simeq 6000 \mathrm{~km} \mathrm{~s}^{-1}$ (Eracleous \& Halpern 1994). Moreover, a broad (FWHM $\simeq$ $\left.1450 \mathrm{~km} \mathrm{~s}^{-1}\right) \mathrm{H}_{\alpha}$ component was detected in polarized flux (Corbett et al. 1998). The interstellar medium should be distributed according to a rather peculiar geometry, for bow shock interactions to produce a broad polarized line.

An interesting suggestion was recently put forward by Bellamy et al. (2003), who discovered broad $\mathrm{Pa} \alpha$ line emission and a reddened continuum in the young radio galaxy PKS 1549-79. The compactness of the radio emission in this object, together with the one-side jet morphology, indicate that the radio axis is probably aligned close to the line-of-sight. According to the 0-th order AGN unification scenarios, the BLR and the AGN in this object should be unobscured, contrary to evidence. Bellamy et al. propose a scenario, whereby 
PKS 1549-79 is an AGN in the early stages of its evolution. The high obscuration should be a transitory phase, which will end as gas and dust are dissipated by outflowing gas. The analogies between PKS 1549-79 and Mkn 668 are remarkable, both exhibiting simultaneously an obscured nucleus, an outflowing BLR, and a small-scale radio jet. However, the radio morphology of Mkn 668 exhibits clearly two radio-lobes, although their power differs by about one order of magnitude (Kameno et al. 2000). This prevents the line-of-sight to the AGN in Mkn 668 from being too close to the radio axis. Moreover, the detection of a large-scale ( $\simeq 30 \mathrm{kpc}$; de Bruyn 1990) radio halo may indicate that Mkn 668 could be undergoing one of several phases of recurrent activity.

Acknowledgements. This paper is based on observations obtained with XMM-Newton, an ESA science mission with instruments and contributions directly funded by ESA Member States and the USA (NASA). This research has made use of data obtained through the High Energy Astrophysics Science Archive Research Center Online Service, provided by the NASA/Goddard Space Flight Center and of the NASA/IPAC Extragalactic Database (NED) which is operated by the Jet Propulsion Laboratory, California Institute of Technology, under contract with the National Aeronautics and Space Administration. M.G. gratefully acknowledges a period of visiting astronomer at the Harvard-Smithsonian Center for Astrophysics, supported by NASA Grant \#NAS8-39073, where most of the research presented in this paper was conducted. Useful discussions with F. Govino are acknowledged.

\section{References}

Antonucci, R. 1993, ARA\&A, 31, 473

Bekar, J. C., Hunstead, R. W., \& Brinkmann, W. 1995, MNRAS, 277, 553

Barone, K. 2003, Un. degree thesis

Barthel, P. D. 1989, ApJ, 336, 606

Bellamy, M. J., Tadhunter, C. N., Morganti, R., et al. 2003, MNRAS, 344, L80

Bianchi, S., Matt, G., \& Iwasawa, K. 2001, MNRAS, 322, 699

Bicknell, G. V., Dopita, M. A., \& O’Dea, C. P. O. 1997, ApJ, 485, 112

Blake, G. M., Argue, A. N., \& Kenworthy, C. M. 1970, ApJ, 6, L167

Boller, T., Keil, R., Hasinger, G., et al. 2003, A\&A, 411, 63

Carvalho, J. C. 1985, MNRAS, 215, 463

Carvalho, J. C. 1998, A\&A, 329, 845

Celotti, A., Ghisellini, G., \& Chiaberge, M. 2001, MNRAS, 321, L1

Celotti, A., Padovani, P., \& Ghisellini, G. 1997, MNRAS, 286, 415

Corbett, E. A., Robinson, A., Axon, D. J., Young, S., \& Hough, J. H. 1998, MNRAS, 296, 721

Dallacasa, D., Stanghellini, C., Centonza, M., \& Fanti, R. 2000, A\&A, 363, 887

de Bruyn, A. G. 1990, in Variability of Active Galaxies, ed. W. J. Duschl, S. J. Wagner, \& M. Camezind (Heidelberg), Lect. Notes Phys., 377, 105

de Vries, W. H., O’Dea, C. P., Baum, S. A., et al. 1998, ApJ, 503, 156 De Young, D. S. 1993, ApJ, 402, 95

Dickey, J. M., \& Lockman, F. J. 1990, ARA\&A, 28, 215

Elvis, M., Fiore, F., Mathur, S., \& Wilkes, B. J. 1994, ApJ, 425, 103

Eracleous, M., \& Halpern, J. P. 1994, ApJS, 90, 1

Evans, I. N., Ford, H. C., Kinney, A. L., et al. 1991, ApJ, 369, L27

Fanti, C., Fanti, R., Dallacasa, D., et al. 1995, A\&A, 302, 317
Ghisellini, G., Padovani, P., Celotti, A., \& Maraschi, L. 1993, ApJ, 407, 65

Ghisellini, G., Haardt, F., \& Matt, G. 1994, MNRAS, 267, 743

Greenhill, L. J., Ellingsen, S. P., Norris, R. P., et al. 1997, ApJ, 474, L103

Greenhill, L. J., Gwinn, C. R., Antonucci, R., \& Barvainis, R. 1996, ApJ, 472, L21

Guainazzi, M., Matt, G., Antonelli, L. A., et al. 1999, MNRAS, 310, 10

Guainazzi, M., Nicastro, F., Fiore, F., et al. 1998, MNRAS, 301, L1

Guainazzi, M., Oosterbroek, T., Antonelli, L. A., \& Matt, G. 2000, A\&A, 364, L80

Guainazzi, M., Stanghellini, C., \& Grandi, P. 2003, MPE Report, 281, 261

Harris, D. E., \& Krawczynski, H. 2002, ApJ, 565, 244

Jansen, F., Lumb, D., Altieri, B., et al. 2001, A\&A, 365, L1

Kameno, S., Horiuchi, S., Shen, Z.-Q., et al. 2000, PASJ, 52, 209

Knapp, G. R., Bies, W. E., \& van Gorkom, J. H. 1990, AJ, 99, 476

Krolik, J. H., Madau, P., \& Życky, P. 1994, ApJ, 420, L57

Leahy, D. A., \& Creighton, J. 1993, MNRAS, 263, 314

Lister, M. L. 2003, ASP Conf. Ser., in press [arXiv: astro-ph/0301332]

Loiseau, N. 2003, User's Guide to the XMM-Newton Science Analysis System, Issue 2.1, (XMM-Newton Science Operation Center: Villafranca del Castillo)

Magdziarz, P., \& Zdziarski, A. A. 1995, MNRAS, 273, 837

Maiolino, R., Salvati, M., Bassani, L., et al. 1998, 338, 781

Maloney, P. R. 1996, in Cygnus A - Study of a Radio Galaxy, ed. C. L. Carilli, \& D. E. Harris (Cambridge: Cambridge University Press), 60

Marziani, P., Sulentic, J. W., Calvani, M., et al. 1993, ApJ, 410, 56

Matt, G., Brandt, W. N., \& Fabian, A. C. 1996, MNRAS, 280, 823

Matt, G., Guainazzi, M., Maiolino, R., et al. 1999a, A\&A, 341, L27

Matt, G., Pompilio, F., \& La Franca, F. 1999b, NewA, 4, 191

Matt, G., Fabian, A. C., Guainazzi, M., et al. 2000, MNRAS, 318, 173

Matt, G., Bianchi, S., Guainazzi, M., et al. 2003a, A\&A, 399, 519

Matt, G., Guainazzi, M., \& Maiolino, R. 2003b, MNRAS, 342, 422

Mazzarella, J. M., Bothum, G. D., \& Boroson, T. A. 1991, AJ, 101, 2034

Mewe, R., Gronenschild, E. H. B. M., \& van der Oord, G. H. J. 1985, A\&AS, 62, 197

Miley, G. 1980, ARA\&A, 18, 165

Molendi, S., Bianchi, S., \& Matt, G. 2003, MNRAS, 343, L1

Mulchaey, J. S., Koratkar, A., Ward, M. J., et al. 1994, ApJ, 436, 586

Nandra, K., George, I. M., Mushotzky, R. F., Turner, T. J., \& Yaqoob, T. 1997, ApJ, 467, 70

Neufeld, D. A., Maloney, P. R., \& Conger, S. 1994, ApJ, 436, L127

O'Dea, C. 1998, PASP, 110, 493

O’Dea, C., de Vries, W. H., Worrall, D. M., Baum, S., \& Koekemoer, A. 2000, AJ, 119, 478

O'Dea, C., de Vries, W. H., Koekemoer, A. M., et al. 2002, AJ, 123, 2333

Pacholcyzk, A. G. 1970, Radio Astrophysics (San Francisco: Freeman)

Phillips, R. B., \& Mutel, R. L. 1982, A\&A, 106, 21

Pihlström, Y. M., Conway, J. E., \& Vermeulen, R. C. 2003, A\&A, 404, 871

Polatidis, A. G., \& Conway, J. E. 2003, PASA, 20, 69

Protassov, R., van Dyk, D. A., Connors, A., Kashyap, V. L., \& Siemiginowska, A. 2002, ApJ, 571, 545

Readhead, A. C. S., Taylor, G. B., Xu, W., et al. 1996, ApJ, 460, 612

Reeves, J. N., \& Turner, M. J. L. 2000, MNRAS, 316, 234

Sambruna, R., Eracleous, M., \& Mushotzky, R. 1999, ApJ, 526, 60 
Sambruna, R., Netzer, H., Kaspi, S., et al. 2001, ApJ, 546, L13

Scheuer, P. A. G. 1974, MNRAS, 166, 513

Siemiginowska, A., Bechtold, J., Aldcroft, T. L., et al. 2002, ApJ, 570, 543

Siemiginowska, A., Stanghellini, C., Brunetti, G., et al. 2003, ApJ, 595,643

Snellen, I. A. G., Lehnert, M. D., Bremer, M. N., \& Schilizzi, R. T. 2002, MNRAS, 337, 981

Stanghellini, C., O’Dea, C. P., Baum, S. A., \& Laurikainen, E. 1993 , ApJS, 88, 1

Stanghellini, C., Dallacasa, D., O'Dea, C., et al. 1996, in Proc. Second Workshop on GigaHertz Peaked-Spectrum and Compact SteepSpectrum Sources, ed. I. A. Snellen, R. T. Schilizzi, H. J. A. Röttgering, \& M. N. Bremer (Leiden: Leiden Obs.), 4

Stanghellini, C., Bondi, M., Dallacasa, D., et al. 1997, A\&A, 318, 376
Stanghellini, C., Liu, X., Dallacasa, D., \& Bondi, M. 2002, NewAR, 46, 287

Strüder, L., Briel, U., Dannerl, K., et al. 2001, A\&A, 365, L18

Tavecchio, F., Maraschi, L., \& Ghisellini, G. 1998, ApJ, 509, 608

Tavecchio, F., Maraschi, L., Sambruna, R. M., \& Megan Urry, C. 2000, ApJ, 544, L23

Turner, T. J., George, I. M., Nandra, K., \& Mushotzky, R. F. 1997, ApJ, 488, 164

Turner, M. J. L., Abbey, A., Arnaud, M., et al. 2001, A\&A, 365, L27

Uttley, P., McHardy, I. M., Papadakis, I. E., Guainazzi, M., \& Fruscione, A. 1999, MNRAS, 307, L6

Zhang, Y. F., \& Marscher, A. P. 1994, in Proc. of the ROSAT Science Symposium, ed. E. Schlegel, \& R. Petre (New York: American Institute of Physics), 406 